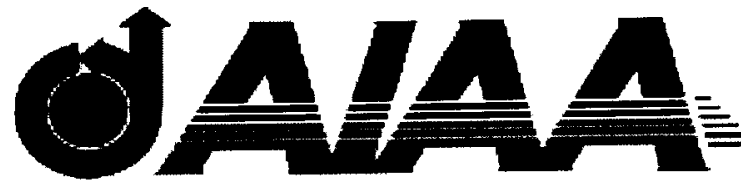

AIAA 2001-2454

\title{
Dynamic Deformation Measurements of an Aeroelastic Semispan Model
}

Sharon S. Graves, Alpheus W. Burner John W. Edwards, and David M. Schuster

NASA Langley Research Center Hampton, VA

$19^{\text {th }}$ AIAA Applied Aerodynamics Conference

$$
\begin{gathered}
\text { June 11-14, } 2001 \\
\text { Anaheim, CA }
\end{gathered}
$$

For permission to copy or republish, contact the American Institute of Aeronautics and Astronautics 1801 Alexander Bell Drive, Suite 500, Reston VA 20191 


\title{
AIAA - $2001-2454$ \\ DYNAMIC DEFORMATION MEASUREMENTS OF AN AEROELASTIC SEMISPAN MODEL
}

\author{
Sharon S. Graves", Alpheus W. Burner", John W. Edwards", \\ and David M. Schuster \\ NASA Langley Research Center, Hampton, VA 23681-2199
}

\begin{abstract}
The techniques used to acquire, reduce, and analyze dynamic deformation measurements of an aeroelastic semispan wind tunnel model are presented. Single-camera, single-view video photogrammetry (also referred to as videogrammetric model deformation, or VMD) was used to determine dynamic aeroelastic deformation of the semispan "Models for Aeroelastic Validation Research Involving Computation" (MAVRIC) model in the Transonic Dynamics Tunnel at the NASA Langley Research Center. Dynamic deformation was determined from optical retroreflective tape targets at 5 semispan locations located on the wing from the root to the tip. Digitized video images from a charge coupled device (CCD) camera were recorded and processed to automatically determine target image plane locations that were then corrected for sensor, lens, and frame grabber spatial errors. Videogrammetric dynamic data were acquired at a $60-\mathrm{Hz}$ rate for time records of up to 6 seconds during portions of this flutter/Limit Cycle Oscillation (LCO) test at Mach numbers from 0.3 to 0.96 . Spectral analysis of the deformation data is used to identify dominant frequencies in the wing motion. The dynamic data will be used to separate aerodynamic and structural effects and to provide time history deflection data for Computational Aeroelasticity code evaluation and validation.
\end{abstract}

\section{INTRODUCTION}

Video photogrammetry was used to measure dynamic deformation on the Models for Aeroelastic Validation Research Involving Computation semispan model (MAVRIC-I), a business jet wing-fuselage flutter model, in NASA Langley's Transonic Dynamics Tunnel (TDT). The overall objective of this test is to provide benchmark validation data on a representative configuration that exhibits nonlinear, transonic aeroelastic response, specifically limit cycle oscillations and buffet onset. Instrumentation included unsteady pressure transducers, accelerometers, and strain gages. Computational aeroelastic analysis will be conducted as part of this research to assess and refine state-of-the-art design tools.

The primary objective of this series of MAVRIC tests was to provide detailed experimental wind-tunnel data suitable for Computational Aeroelasticity (CAE) code evaluation and validation at transonic separation onset conditions. Unsteady pressures and wing responses were obtained for three wingtip configurations: clean,

- Research Engineer, Member AIAA.

${ }^{\dagger}$ Research Engineer, Senior Member AIAA.

† Senior Research Engineer, Fellow AIAA.

Senior Research Engineer, Associate Fellow AIAA. Copyright 2001 by the American Institute of Aeronautics and Astronautics, Inc. No copyright is asserted in the United States under Title 17, U.S. Code.
The U.S. Government has a royalty-free license to exercise all rights under the copyright claimed herein for Governmental Purposes. All other rights are reserved by the copyright owner.

tipstore, and winglet. Traditional flutter boundaries were measured over the range of $\mathrm{M}=0.6$ to 0.9 and maps of Limit Cycle Oscillation (LCO) behavior were made in the range of $\mathrm{M}=0.85$ to 0.95 . The dynamic pressure transducers provide time histories of the pressure distribution on the wing as it encounters the flutter or LCO phenomena. However, these pressures are directly dependent on the motion of the wing. Accurate measurement of the wing motion is a critical item when comparing the unsteady surface pressures with computed results. Modern computational aeroelasticity programs are capable of simultaneously computing both the vehicle motion and dynamic loads on the vehicle. However, accurate simultaneous computation of these components is difficult for highly nonlinear problems such as $\mathrm{LCO}^{1,2}$ and it is very beneficial to be able to isolate the various components of the problem. This is where the videogrammetry data is of greatest use. Specifying the model motion using data obtained through the videogrammetric system, the issue of computing the model motion can be eliminated from the computational problem, and a direct comparison of computed and wind tunnel pressures can be performed. Researchers previously depended on strain gage and accelerometer data to estimate the wing 
motion. Videogrammetry provides a significantly more accurate and direct method for obtaining these data.

The intent of this paper is to relate experiences using the videogrammetry technique in a large production wind tunnel for dynamic deformation measurements in order to aid potential users of the technique at the TDT and other facilities. Rather than presenting extensive deformation data, only representative data will be included. The data acquisition procedure and interaction with the facility data acquisition system will be described. This work is part of an overall effort to develop a dynamic model deformation measurement capability up to $1000 \mathrm{~Hz}$.

\section{MODEL}

The MAVRIC-I Business Jet model, shown in Figure 1,

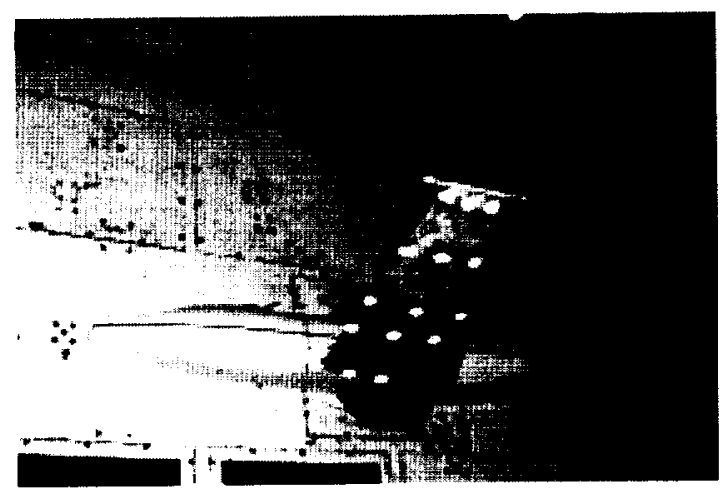

Figure 1 MAVRIC-I model mounted on tunnel sidewall.

consists of a structurally flexible wing mounted low on a rigid fuselage. The semi-span model is constructed of a stepped thickness aluminum plate planform and covered with end-grain balsa wood to provide the wing contour. The wing, with no twist or dihedral, was designed to flutter in the TDT at dynamic pressures between 100 and 200 pounds per square foot. Initial testing of this model in the early 1990's verified the flutter characteristics of the wing, and in the process, also uncovered large-amplitude nonlinear dynamic response behavior. This Limit Cycle Oscillation (LCO) occurs in the transonic flight regime well below the wing's flutter boundary and is similar to nonlinear phenomena encountered on some operational aircraft such as the F-16. To further investigate this nonlinearity, the model instrumentation was significantly upgraded by adding 87 in situ dynamic pressure transducers, eight accelerometers buried in the wing at four spanwise locations, and incorporating the videogrammetric deformation system to measure the dynamic motion of the model
This new instrumentation complements the original instrumentation suite that included wing root-bending and torsion strain gages and wingtip accelerometers. The model was thoroughly retested in the TDT and detailed measurements were acquired at numerous flutter and LCO conditions. ${ }^{4}$ Figure 2 shows the three wing-tip configurations used: clean wingtip (body of

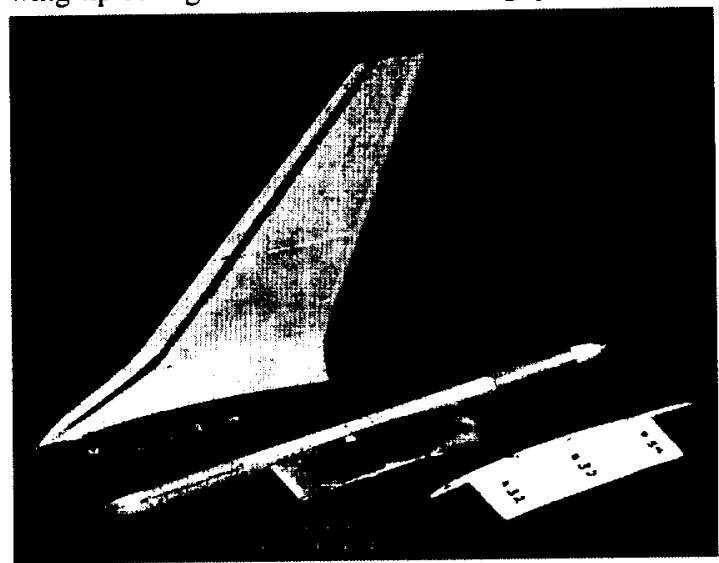

Figure 2 Wingtip configurations: clean wingtip, pencil tipstore, winglet.

revolution), pencil tipstore, and winglet with a 41 -

degree leading-edge sweep.

TRANSONIC DYNAMICS TUNNEL

The Langley Transonic Dynamics Tunnel (TDT) is a unique national facility dedicated to identifying, understanding, and solving aeroelastic problems. The TDT is specifically dedicated to investigating flutter problems of fixed-wing aircraft. The tunnel is also used to investigate other aeroelastic phenomena such as fixed-wing buffet and divergence. The tunnel is used for studying the use of active controls technologies for both fixed-wing and rotary-wing configurations, clearing new designs from flutter, determining the effects of ground-wind loads on launch vehicles, and providing steady and unsteady aerodynamic pressure data to support computational aeroelasticity and additional fluid dynamics code development and validation. Dynamic deformation data can be very useful in these studies, especially if the measurements have little negative impact on facility productivity. The TDT is a closed-circuit, continuous flow, variable pressure wind tunnel with a $16-\mathrm{ft}$ square test section with cropped corners. The tunnel is capable of testing at stagnation pressures from near zero to atmospheric conditions. Controlled variation of pressure in the tunnel is used to simulate variations in flight altitude. The tunnel is capable of using either air or R-134a as the test medium. Testing in a heavy gas, such as $R-$ $134 \mathrm{a}$ has important advantages over testing in air, particularly for aeroelastic models. The R-134a 
refrigerant is about 4 times as dense as air, yet has a speed of sound of about half that of air. These advantages include improved model to full-scale similitude, higher Reynolds numbers, easier fabrication of scaled models, reduced tunnel power requirements, and in the case of rotary-wing models, reduced model power requirements. The tunnel can operate up to a Mach number of 1.2 and is capable of maximum Reynolds numbers of about $3 \times 10^{6}$ per $\mathrm{ft}$ in air and 10 $x 10^{6}$ per $\mathrm{ft}$ in $\mathrm{R}-134 \mathrm{a}$.

\section{VIDEOGRAMMETRIC MEASUREMENT TECHNIOUE}

The videogrammetric measurement technique is an optical method characterized by automated image processing, sub-pixel resolution, near routine, near realtime measurements, and high data volume with minimum impact to productivity. ${ }^{3,5,6,7}$ The technique consists of a single-camera, single-view, photogrammetric solution from digital images of targets placed on the wing at known semi-span locations. Except for the targets, the technique is non-intrusive. For this application the thickness of the retro-reflective tape targets (0.1 millimeters) was estimated to have negligible effect on the aeroelastic behavior based on the nearly identical pressure data obtained with targets on as with targets off. When a light source is positioned near the camera the light retro-reflected from the tape targets can greatly exceed that possible with white diffuse targets, resulting in a high contrast image in which the targets are easily discriminated from the background. Such high contrast images are amenable to automated image processing.

\begin{tabular}{|c|cc||}
\hline$\eta$ & $Y$, in & $D$, in \\
\hline \hline 0.0916 & 4.87 & 1.5 \\
\hline 0.3513 & 18.68 & 1.25 \\
\hline 0.4977 & 26.46 & 1.25 \\
\hline 0.7522 & 39.99 & 1.25 \\
\hline 0.9656 & 51.34 & 1.0 \\
\hline
\end{tabular}

Table I. Semi-span, $\eta$, target positions and diameters.

The basic hardware consists of an instrumentationgrade video-rate Hitachi KP-Ml CCD camera, an Epix frame grabber board, and a Pentium IIl-based computer with image acquisition and reduction software. A fiber optic light source was located as close to the camera as possible to take advantage of the retro-reflective nature of the optical targets. The camera was positioned to the side and below the model, resulting in an oblique view of the model at an angle of about $32^{\circ}$ to the horizontal. Targets with diameters, $D$, of 1.0 to 1.5 inches were placed in rows ( 3 targets per row) at 5 known semispan locations, $\eta$, from near the wing root to near the wing tip (Table I).

Acquisition and digitization of a live video stream at a nominal $60 \mathrm{~Hz}$ rate was triggered by the facility Data Acquisition System (DAS). Once the video sequence was acquired, a blob analysis was used for target detection in the image. A gray-scale centroid calculation with the background level automatically removed provided sub-pixel accuracy. Single-view photogrammetry was then used to determine the $X$ (stream-wise) and $Z$ (vertical) coordinates in object space, given the known $Y$ (cross-flow) coordinates. $Z$ intercept and slope angles were computed by a linear least squares fit in $X-Z$ space for each $\eta$-station along the wing.

\section{CAMERA CALIBRATION}

Camera calibration consists of determining image plane correction parameters for lens distortion and lens alignment to the CCD sensor as well as the determination of the location and pointing angles of the camera in the test section coordinate system. The parameters for image plane corrections were determined in a laboratory prior to setting up the measurement system in the test section at the TDT.

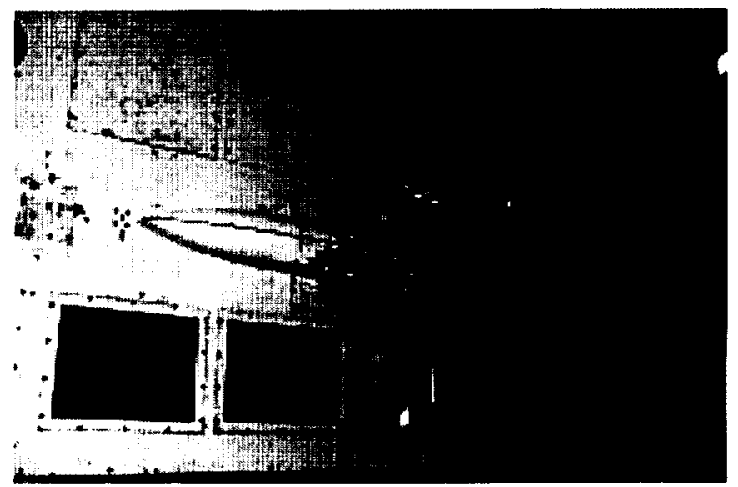

Figure 3 Calibration plate on model wingtip.

A calibration fixture consisting of a number of targets with known spatial coordinates was used to determine lens distortion and principal distance (Figure 3). Only third order radial distortion and a single term for decentering distortion were found to be statistically significant to warrant their inclusion in the correction parameters. A laser illumination technique was used to determine the photogrammetric principal point and point of symmetry for distortion. Once the measurement system was set up with the proper view of the model a calibration fixture was aligned to the test section coordinate system in order to determine the 
pointing angles and location of the camera via photogrammetric space resection.

\section{DATA REDUCTION}

The VMD measurement technique uses photogrammetry to extract two-dimensional (2D) images and map them into a three dimensional (3D) object space. The collinearity equations provide a mapping of coordinates between 3D object space and the $2 \mathrm{D}$ coordinates in the image plane. The $n$th target location point $p_{n}=\left(x_{n}, y_{n}\right\}^{T}$ in the image plane is related to a point $P_{n}=\left(X_{n}, Y_{n}, Z_{n}\right\}^{T}$ in the object space by

$$
\begin{aligned}
& x_{n}-x_{p}+d x=-c \frac{m_{11}\left(X_{n}-X_{c}\right)+m_{22}\left(Y_{n}-Y_{c}\right)+m_{33}\left(Z_{n}-Z_{c}\right)}{m_{31}\left(X_{n}-X_{c}\right)+m_{32}\left(Y_{n}-Y_{c}\right)+m_{33}\left(Z_{n}-Z_{c}\right)} \\
& y_{n}-y_{p}+d y=-c \frac{m_{21}\left(X_{n}-X_{c}\right)+m_{22}\left(Y_{n}-Y_{c}\right)+m_{23}\left(Z_{n}-Z_{c}\right)}{m_{31}\left(X_{n}-X_{c}\right)+m_{32}\left(Y_{n}-Y_{c}\right)+m_{3}\left(Z_{n}-Z_{c}\right)}
\end{aligned}
$$

The interior orientation of the camera is given by the parameter set $\left(c, x_{p}, y_{p}\right)$. The exterior orientation of the camera is given by the parameter set $\left(\omega, \phi, \kappa, X_{c}, Y_{c}, Z_{c}\right)$, where $\omega, \phi, \kappa$ are rotational Euler angles and $X_{c}, Y_{c}, Z_{c}$ represent the coordinates of the perspective center of the camera in object space. The $d x$ and $d y$ terms are due to lens distortion. The elements of the rotation matrix in the above equations are given by

$$
\begin{aligned}
& m_{11}=\cos \phi \cos \kappa \\
& m_{12}=\sin \omega \sin \phi \cos \kappa+\cos \omega \sin \kappa \\
& m_{13}=-\cos \omega \sin \phi \cos \kappa+\sin \omega \sin \kappa \\
& m_{21}=-\cos \phi \sin \kappa \\
& m_{22}=-\sin \omega \sin \phi \sin \kappa+\cos \omega \cos \kappa \\
& m_{23}=\cos \omega \sin \phi \sin \kappa+\sin \omega \cos \kappa \\
& m_{31}=\sin \phi \\
& m_{32}=-\sin \omega \cos \phi \\
& m_{33}=\cos \omega \cos \phi
\end{aligned}
$$

The local angle, $\alpha_{1}$ at each $\eta$-station is defined by

$$
\alpha_{l}=-\tan ^{-1}(\Delta Z / \Delta X)
$$

Local wing twist, $\theta$, due to aerodynamic loads is defined as ${ }^{7}$

$$
\theta=\alpha_{l}^{o n}(\eta)-\alpha_{l}^{o f}(\eta)
$$

where $\alpha_{l}^{o n}(\eta)$ and $\alpha_{l}^{\text {off }}(\eta)$ are the local angles, $\alpha_{l,}$ in the wind-on and wind-off cases at the same semispan location $\eta$.

In a single camera VMD system, a solution $(X, Y, Z)$ to the collinearity equations from a single set of image coordinates $(x, y)$ is not possible unless additional information is provided. Since motion was basically confined to the pitch plane during this test, the spanwise locations $(Y)$ of the targets are fixed so that the number of unknowns reduces to $X$ and $Z$.

\section{RESULTS}

The objective of this evaluation test was to assess the value of videogrammetric data during flutter and limit cycle oscillation (LCO) testing and to determine operational characteristics and capabilities that might prove valuable in future measurement systems. A secondary objective was to obtain additional experience with videogrammetric techniques for dynamic measurements as part of an overall program to develop a videogrammetric dynamic (up to $1000 \mathrm{~Hz}$ ) measurement capability. For all tests with the videogrammetric system in the past the primary objective has been the measurement of static model and component deformation, not flutter and LCO dynamic measurements.

Set up and calibration of the videogrammetric system occurred near the end of the MAVRIC test due to the unscheduled piggy-back nature of this evaluation experiment. For this reason, as well as the very time critical nature of flutter testing, the videogrammetric measurement system had a low priority. Thus a number of points were missed because another data point was taken before the data system had finished processing all the images of a sequence and could not respond to a trigger from the tunnel DAS. It is expected that the loss of data points during time critical testing will not be a major issue with a new, much faster measurement system currently undergoing development at NASA Langley. ${ }^{8}$. Some data points were also lost due to the overheating of the light source caused by low tunnel pressure that reduced significantly the amount of convection cooling possible. When the power supply reached a certain temperature the light source would be automatically powered down by a thermal relay and 
could not be restarted until the temperature had dropped sufficiently. Once this problem was diagnosed the light was remotely cut off after each data point to lessen the only 2 seconds ( 120 images) of data were taken in order to reduce the time between points, but with reduced fidelity of the temporal recordings.
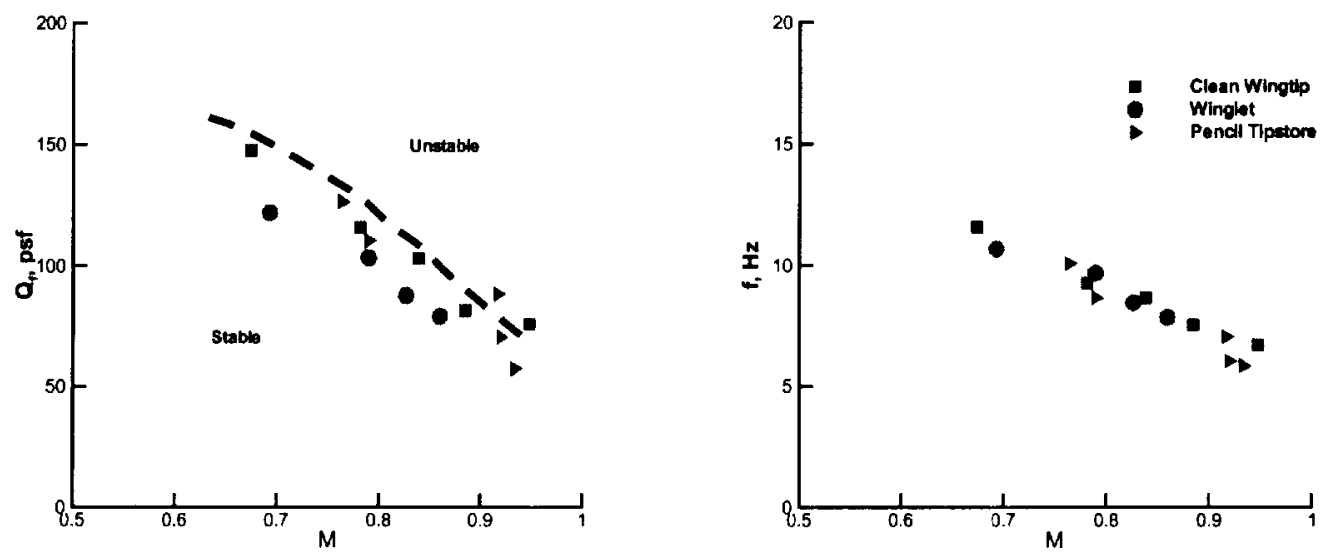

Figure 4 Test points at approximate flutter boundary (dashed line) and frequencies in heavy gas.

chances of over heating and the loss of data for the next point. This procedure however would sometimes lead to a missed point if the videogrammetric measurement system operator was not aware that a point was about to be taken and failed to turn the light source back on in time. Developments are currently underway on LED array light sources that offer the promise of high output and additional synchronization options, that will be relatively immune to overheating due to low tunnel pressure.

For the portion of the test during which the videogrammetric system was operated, data were taken over a dynamic pressure $(q)$ range of 30 to $150 \mathrm{psf}$, a Mach number $(M)$ range of 0.6 to 0.96 and at nominal model pitch angles $(\alpha)$ of $-0.4,0.6,1.6$, and 2.0 degrees in heavy gas (R134a) mode only. Most of the videogrammetric data were acquired at $\alpha=0.6^{\circ}$, thus results presented here are mainly for that model pitch angle. Measurements were made with three wing tips: clean, pencil tipstore, and winglet. Initially 6-second records at 60 images per second (yielding 360 images per point) were acquired at each data point. The time to fully process 360 images was typically 2 minutes and 20 seconds. The major portion of the processing time was expended in the image processing to extract centroid image plane coordinates of the 15 target locations for each image. The portion of the automated data reduction to convert from pixels to units of length (via photogrammetry) took only a few seconds. Later on in the test the time records were reduced to 5 seconds (300 images). For some selected data points
The fully automated data results were output to several text data files. All the files have header information to fully describe the data columns. An append file with $X$, $Y, Z$ mean coordinates for the 15 targets on the wing for each data point served as a summary data file for the mean coordinates. A text data file was also created for each data point that contained the $X, Y, Z$ data as a function of time for all 15 targets for that particular data point. Note that the $Y$-value in these files is not computed, but is based upon known target locations. Another file was created that contained the wing twist angle and vertical $Z$ displacement for each of the 5 rows of targets as a function of time. Still another file was created that recorded tunnel data via a network data link for each data point recorded by the videogrammetric system.

Summary plots of the dynamic pressure-Mach number test space for $\alpha=0.6^{\circ}$ data are presented in Figures 4 through 8. Data for the three tips, clean, pencil tipstore, and winglet, are indicated with square, triangle, and circle symbols respectively. The approximate flutter boundary and near-by data points representing the maximum $q$ and $M$ where near flutter-like behavior occurs is illustrated with a dashed line in Figure 4. Note that for flutter testing, tunnel pressure is held constant and the test section Mach number and dynamic pressure are increased together by slow increases in the wind tunnel fan speed. For flutter/LCO testing the data points are generally started reasonably far from the estimated flutter boundary with $q$ and $M$ being slowly and cautiously increased (and roughly perpendicularly 
to the boundary) toward the flutter boundary. The arrow on Figure 9 illustrates the path of the $q-M$ sweep toward the flutter boundary for 3 such cases. As the

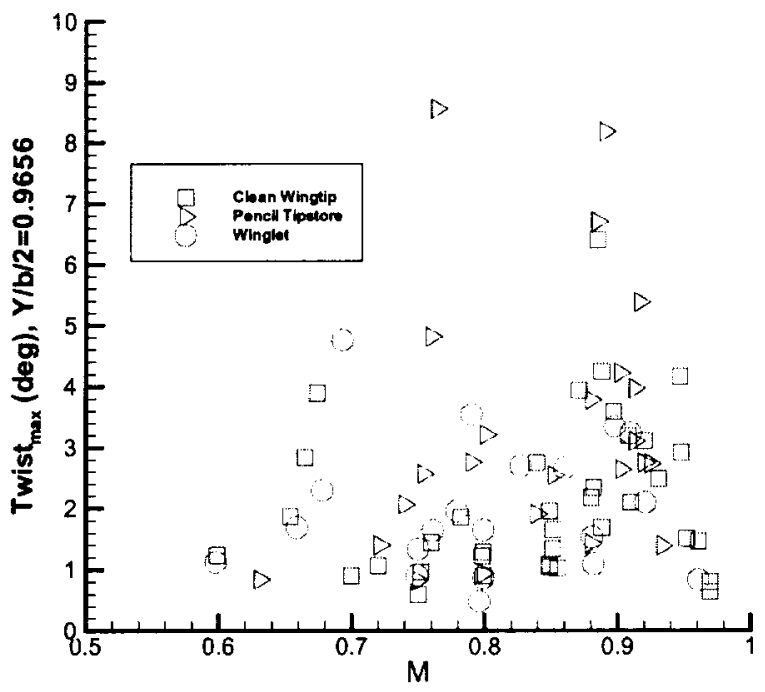

Figure 4 Maximum twist versus $M$ at $\eta=0.9656$

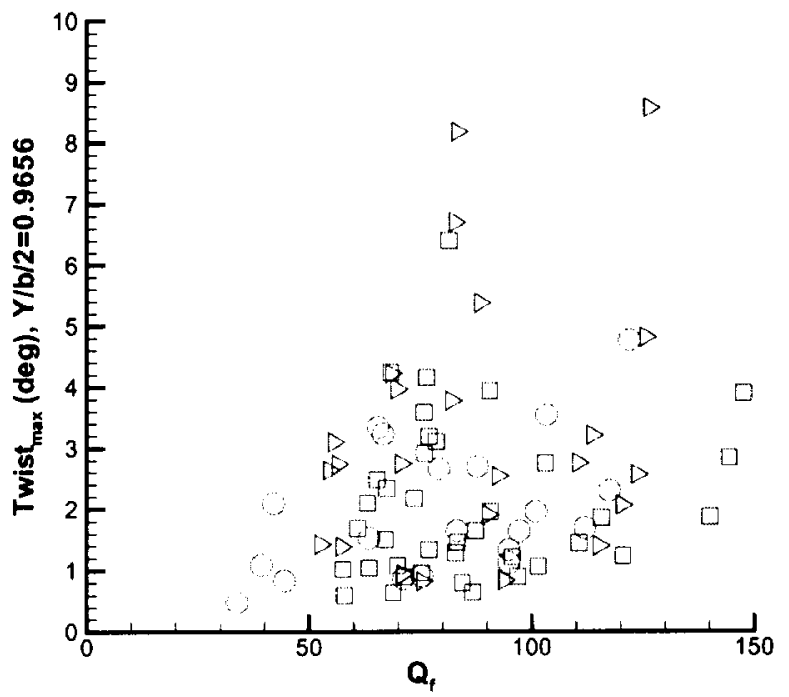

Figure 6 Maximum twist versus $Q$ at $\eta=0.9656$

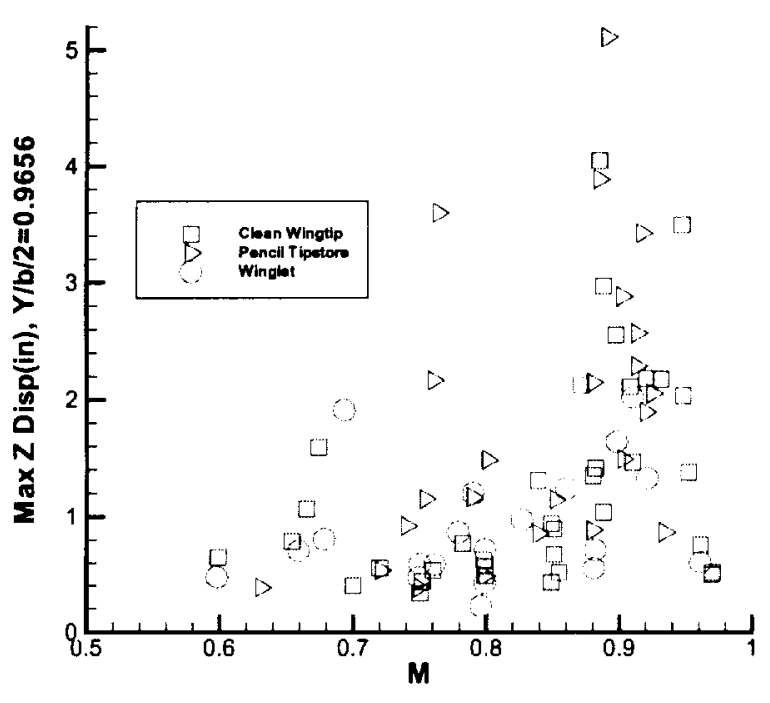

Figure 7 Maximum $\Delta Z$ versus $M$ at $\eta=0.9656$

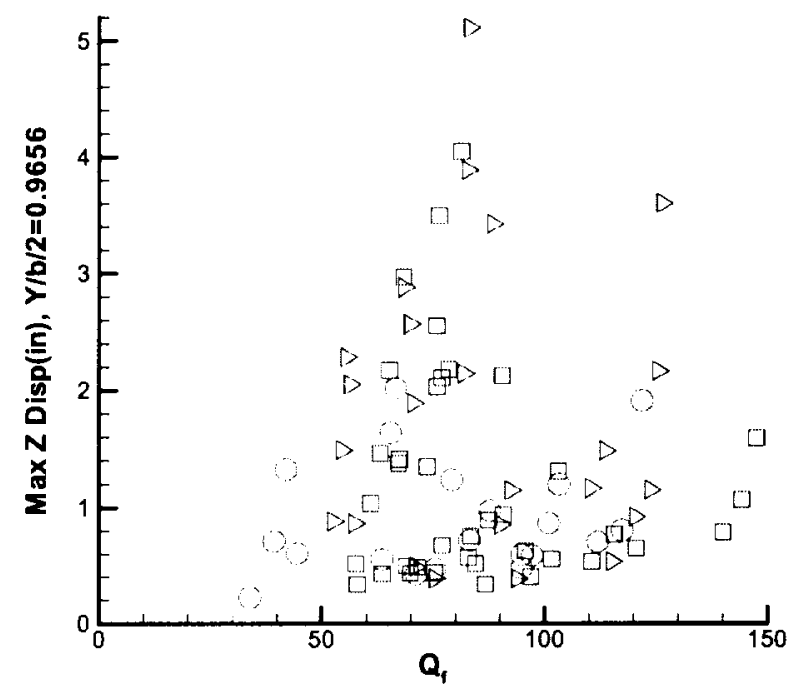

Figure 8 Maximum $\Delta Z$ versus $Q$ at $\eta=0.9656$ 


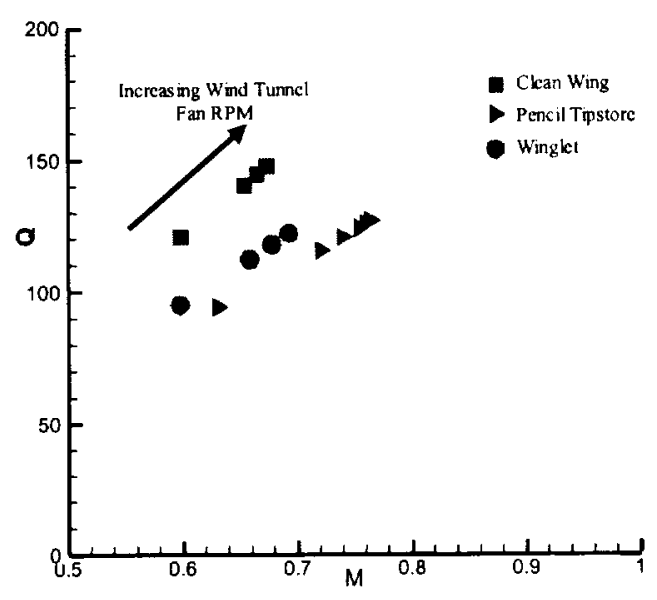

Figure 9 Example of test points approaching flutter boundary.

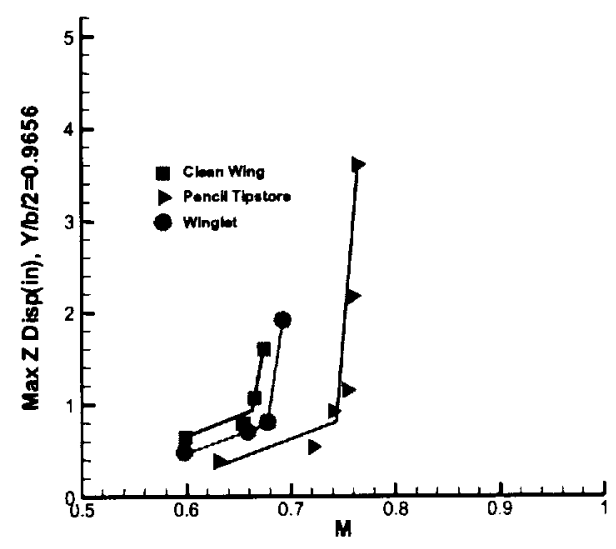

Figure $10 \mathrm{Max} \Delta \mathrm{Z}$ vs $\mathrm{M}$

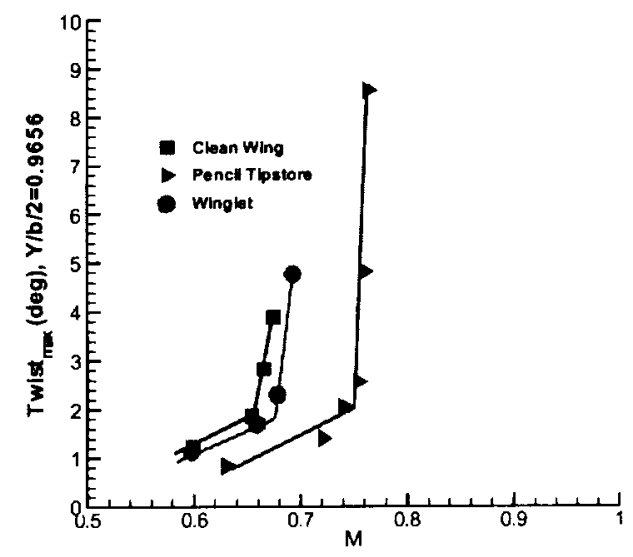

Figure $11 \mathrm{Max} \Delta$ Twist versus $\mathrm{M}$ testing conditions reach the flutter boundary excessive acceleration of the model is noted and a by-pass switch can be manually thrown by an experienced operator to quickly reduce tunnel conditions to below the point where flutter occurs in order to prevent damage to the model. However the videogrammetric system was not automatically triggered by the bypass switch, thus no data were taken at by-pass points and all data points at by-pass are missing. It is expected that future versions of the measurement system will offer post trigger options in order to allow for the possibility to acquire data for a reasonable amount of time before and after the by-pass switch is thrown. Such temporal records slightly before, during, and after by-pass may prove valuable for future flutter/LCO tests.

Data summary plots for the $\alpha=0.6^{\circ}$ data giving the maximum change in twist in degrees at $\eta=0.966$ are presented in Figure 5 as a function of $M$ and in Figure 6 as a function of $q$. Again, data for the three tips, clean, pencil tipstore, and winglet, are indicated with square, triangle, and circle symbols respectively. The maximum peak-to-peak change in twist approached nearly $9^{\circ}$ for one of the data points that happened to be taken especially near to the flutter boundary. The maximum $\Delta Z$ in inches at $\eta=0.966$ is presented in Figure 7 as a function of $M$ and in Figure 8 as a function of $q$.

The videogrammetric system may be used as an indicator of onset of the flutter boundary to complement accelerometers that were placed near the tip at the leading and trailing edges of the wing. Plots of peakto-peak $\Delta Z$ and $\Delta \theta$ are shown in figures 10 and 11 versus $M$ for the point sequences plotted in figure 9 . The rise in $\Delta \theta$ and $\Delta Z$ as $M$ (and correspondingly $q$ ) is increased toward the flutter boundary may be useful in a future real-time implementation of the videogrammetric technique to assist a trained operator or eventually even become part of an automatic by-pass detection system.

Plots of twist and bending with peak-to-peak $\Delta Z$ and $\Delta \theta$ as error bars are shown in Figure 12 for points near and far from the flutter boundary. Five wind-off points made over several days are also included in the plots. The mean wind-off data were subtracted from the windon data to obtain the twist and bending due to aerodynamic load.

The frequency of the variation of $\Delta Z$ and corresponding $\Delta \theta$ versus time of the five target rows for a typical data point near the flutter boundary are plotted in Figure 13. The frequency spectra were calculated by Fast Fourier Transforms (FFT) of the 5 second records of each $\Delta \theta$ 


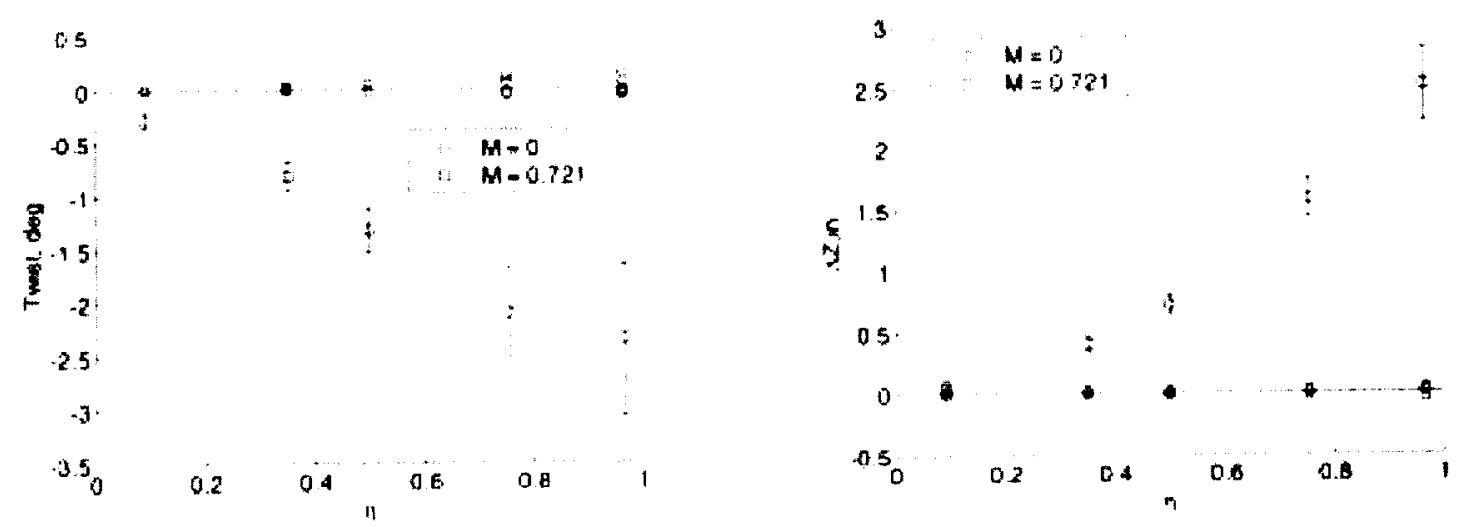

(a) far from flutter boundary

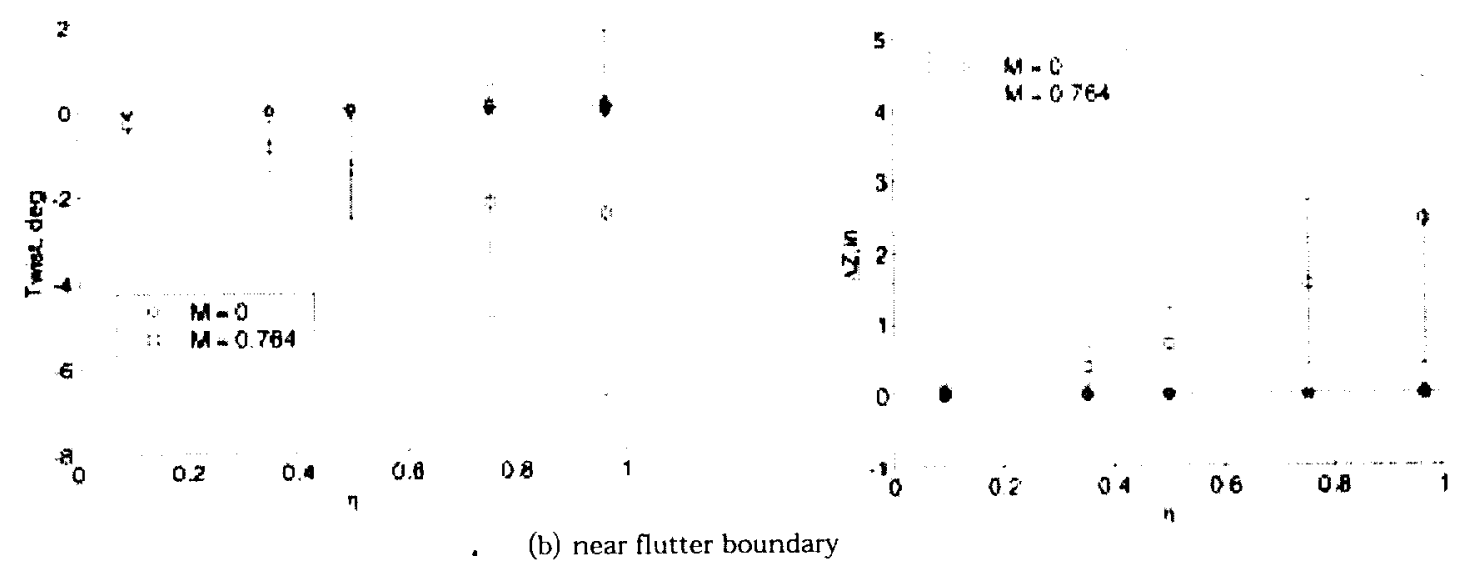

Figure 12 Twist and bending with peak-to-peak $\Delta Z$ and $\Delta \theta$ as error bars are shown for points near and far from flutter boundary 

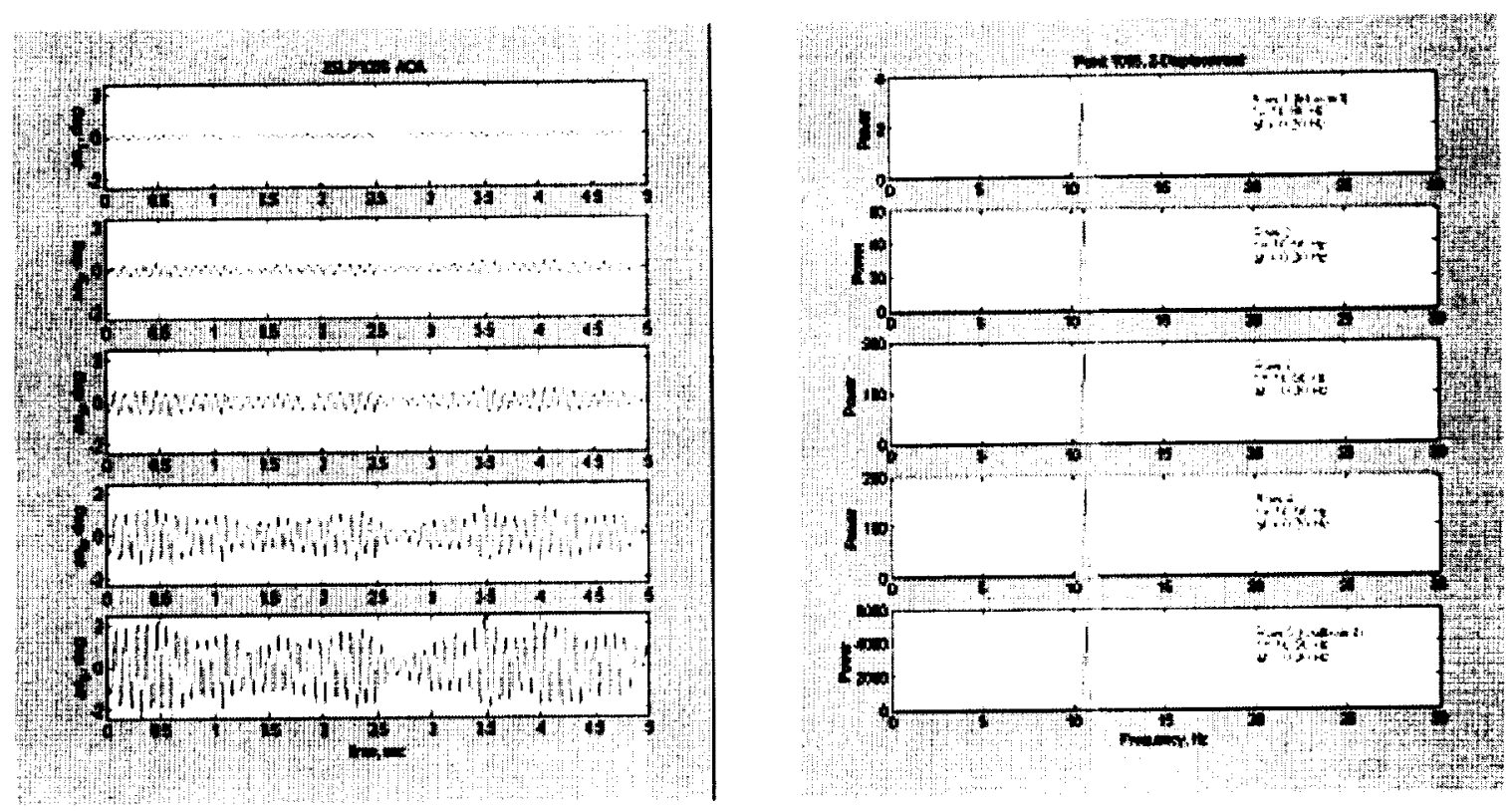

Figure 13 Frequencies for 5 rows of targets and corresponding $\Delta \theta$ versus time

and $\Delta Z$ time history. Generally it was found that the frequencies of all 5 rows on the wing from inboard at $\eta$ $=0.0916$ to outboard at $\eta=0.966$ were equal. The Nyquist frequency for these samples was $29.97 \mathrm{~Hz}$ based on a sampling frequency of $59.94 \mathrm{~Hz}$. The value
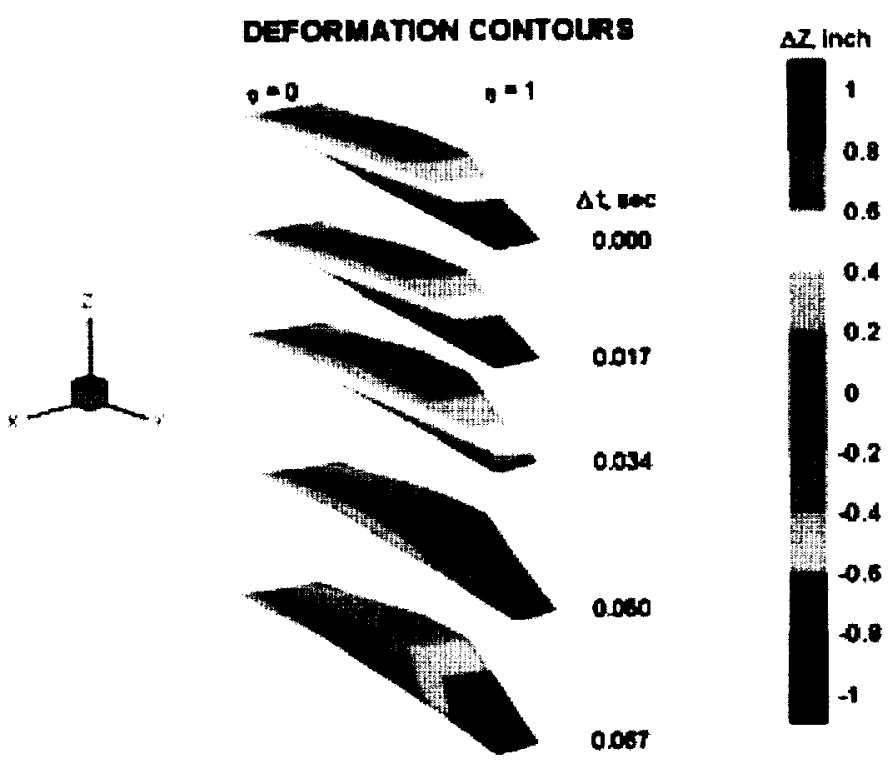

0.007
$59.94 \mathrm{~Hz}$ instead of $60 \mathrm{~Hz}$ is derived from the standard pixel clock frequency of $14.31818 \mathrm{MHz}$ common to RS-170 cameras. (The use of $59.94 \mathrm{~Hz}$ sampling instead of $60 \mathrm{~Hz}$ can sometimes lead to low frequency beating in gray scale between the light source at a nominal $60 \mathrm{~Hz}$ frequency and the camera frequency.) The spectral resolution was $0.20 \mathrm{~Hz}$ and 0.17 $\mathrm{Hz}$ for the 5 and 6 second records respectively. Dynamic deformation contours for the first five images in a sequence are plotted in Figure 14.

Comparisons of half-amplitude determined with the videogrammetric measurement system at $\eta=0.966$ and with accelerometer data at $\eta=0.90$ are given in Table II. Differences in the measured data can be accounted for because the accelerometer and the videogrammetric measurement system were not at identical locations. The maximum displacement $\left(D_{\max }\right)$ of the accelerometer data was

Figure 14 Deformation contours for the first five images in a sequence 
computed assuming sinusoidal oscillations from

$$
D_{\max }=\frac{a}{4 \pi^{2} f^{2}}
$$

where $a$ is the acceleration and $f$ is the frequency.

\begin{tabular}{|c|ccc|}
\hline$D_{\max } A c c$, in & $D_{\max } V i d$, in & $q, \mathrm{psf}$ & $M$ \\
\hline 0.82 & 0.58 & 123.6 & 0.754 \\
\hline 0.89 & 1.08 & 125.4 & 0.760 \\
\hline 1.68 & 1.80 & 126.4 & 0.764 \\
\hline
\end{tabular}

Table II. $D_{\max }$ as determined by accelerometer and videogrammetric data for dynamic pressure, $q$, and Mach number, $M$.

\section{CONCLUSIONS}

Accurate measurement of the wing motion during flutter and limit cycle oscillation (LCO) testing is critical when comparing unsteady surface pressures with computed results. Modern computational aeroelasticity programs are capable of simultaneously computing both the vehicle motion and dynamic loads on the vehicle. However, accurate simultaneous computation of these components is difficult for highly nonlinear problems such as LCO and it is very beneficial to be able to isolate the various components of the problem. By specifying the model motion using data obtained with videogrammetry, the issue of computing the model motion can be eliminated from the computational problem, and a direct comparison of computed and wind tunnel pressures can be performed. Videogrammetry provides a significantly more accurate and direct method for obtaining these crucial data and appears to be a very useful complement to accelerometer data for future flutter and limit cycle oscillation (LCO) testing.

\section{REFERENCES}

1. Bunton, R. W. and Denegri, C. M. Jr.: Limit Cycle Oscillation Characteristics of Fighter Aircraft. Journal of Aircraft, Vol. 37, No. 5, September-October 2000, pp. 916-918.

2. Denegri, C. M. Jr.: Limit Cycle Oscillation Flight Test Results of a Fighter with External Stores. Journal of Aircraft, Vol. 37, No. 5, September-October 2000, pp. 761-769.

3. Burner, A. W.: Model Deformation Measurements at NASA Langley Research Center. Published in AGARD (NATO) CP 601 (papers presented at the 81st Fluid Dynamics Panel Symposium on Advanced Aerodynamics Measurement Technology, 22-25 September, 1997, Seattle, Washington) pp 34-1 to 34-9, May 1998.

4. Edwards, J.W., Schuster, D. M., Spain, C. V., Keller, D. F. and Moses, R. W., MAVRIC Flutter Model Transonic Limit Cycle Oscillation Test, AIAA 20011291, April, 2001

5. Liu, T., Radeztsky, R., Garg. S. and Cattafesta, L., "A Videogrammetric Model Deformation System and its Integration with Pressure Paint", AIAA paper 99. $0568,37^{\text {th }}$ AIAA Aerospace Sciences Meeting, Jan 11 $14,1999$.

6. Burner, A. W., Liu, A., Garg, S., Ghee, T., and Taylor, N., "Aeroelastic Deformation Measurements of Flap, Gap, and Overhang on a Semispan Model", AIAA 2000-2386, $21^{\text {st }}$ AIAA Aerodynamic Measurement Technology and Ground Testing Conference, June 1922,2000 .

7. Burner, A., Wahls, R., and Goad, W., "Wing Twist Measurements at the National Transonic Facility", NASA Technical Memorandum 110229, Feb. 1996.

8. Graves, S. S., Burner, A. W., "Development of an Intelligent Videogrammetric Wind Tunnel Measurement System", to be presented SPIE Optical Diagnostics for Fluids, Solids and Combustion, San Diego, Aug. 2001. 


\section{NASA STI Acquisitions DAA Authorization}

The following papers (copies enclosed) have been DAA approved as Unclassified, Publicly Available documents:

\section{Meeting Presentations:}

11 th ARM Science Team Meeting Proceedings, 3/19-23/2001, Atlanta, GA:

M.M. Khaiyer, et al.: A 3-Year Climatology of Cloud and Radiative Properties Derived...

A.D. Rapp, et al:: Comparison of Shortwave Cloud Radiative Forcing Derived From...

M.L. Nordeen, et al:: GMS-5 Satellite-Derived Cloud Properties Over the Tropical...

V. Chakrapani, et al.: Improvements in AVHRR Daytime Cloud Detection Over the...

15th AIAA Computational Fluid Dynamics Conf., 6/11-14/2001, Anaheim, CA:

B. Diskin, et al: Distributed Relaxation for Conservation Discretizations.

H.L. Atkins, et al:: Analysis of Preconditioning and Relaxation Operators for the...

19th AIAA Applied Aerodynamics Conference, 6/11-14/2001, Anaheim, CA:

S.S. Graves, et al.: Dynamic Deformation Measurements of an Aeroelastic Semispan...

37th AIAA/ASME/ASE/ASEE Joint Proplus. Conf. \& Exhib., 7/9-11/2001, Salt Lake City, UT:

R.C. Rogers, et al: Scramjet Tests in Shock Tunnel at Flight Mach 7, 10 and 15 Conditions.

International Symp. on Shock Waves, 23rd, 7/22-27/2001, Ft. Worth, TX:

R.O. Foelsche, et al.: Hypervelocity Capability of the HYPULSE Shock-Expansion...

AIAA Guidance, Navigation, \& Control Conf. \& Exhibit, 8/6-9/2001, Montreal, Canada:

S. Mondoloni, et al.: An Airborne Conflict Resolution Approach Using A Genetic...

\section{Journal Article:}

J.W. Wilson, et al.: Approach and Issue Relating to Shield Material...(Mat'ls \& Desgn, 2001 ). X. Dong, et al.: Arctic Stratus Cloud Properties and Their Effect on the...(JOG 2001).

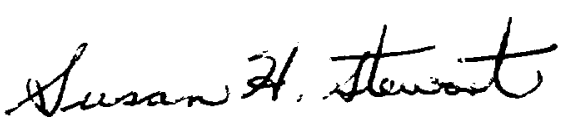

Susan H. Stewart

DAA Representative

NASA Langley Research Center

Mail Stop 196

s.h.stewart@larc.nasa.gov

phone: (757) 864-2518

Hampton, VA 23681-2199 